\title{
The effects of intrauterine ethanol exposure on the levels of Iron and Copper in Cerebrum and Cerebellum of neonatal Wistar rats
}

\author{
${ }^{1,2}$ Ibrahim Shehu El-ladan, ${ }^{1,2}$ Affan Usman \\ ${ }^{1}$ Department of Human Anatomy, \\ Umaru Musa Yar'adua University, \\ Katsina, Dutsinma Road, \\ Katsina, Nigeria \\ ${ }^{2}$ Department of Forensic Science, \\ School of Basic and Applied Sciences, \\ Galgotias University, \\ Uttar Pradesh, India
}

Email: ibrahim.shehu@umyu.edu.ng

\begin{abstract}
Neurodevelopmental disorders have been reported to be associated with infants exposed to ethanol in utero. The study was aimed at evaluating the effects of intrauterine ethanol exposure on neurobehaviour and the amount of iron and copper in the cerebrum and cerebellum of neonatal Wistar rats at different periods of development. Fourteen (14) female Wistar rats were mated with matured males in ratio 2:1 overnight following determination of oestrous phase. Pregnant dams were divided into 7 groups. Group A served as the control that received distilled water. Groups B, C and D were administered $0.5 \mathrm{ml}$ of $20 \%$ ethanol equivalent to $1^{\text {st }}, 1^{\text {st }}$ and $2^{\text {nd }}$ trimesters and whole gestation period (i.e $1^{\text {st }}, 2^{\text {nd }}$ and $3^{\text {rd }}$ trimesters) respectively. Groups $E, F$ and $G$ were given $0.5 \mathrm{ml}$ of $30 \%$ ethanol accordingly. Following parturition, neurobehavioural assessment on sensory and motor reflexes of the litters were tested on postnatal days 5, 6 and 7. Brain tissues were later excised, homogenised and analysed using Atomic Absorption Spectrophotometry. SPSS V20 was used to compare the mean difference using analysis of variance (ANOVA). The ethanol treated neonates in Groups B, E, F and $G$ showed a statistically significant $(p<0.05)$ increase in latency to respond to sensory and motor reflexes when compared with Control Group. Interrelated elevation of both iron and copper was observed in the cerebellum while both the amounts of iron and copper in the cerebrum were depleted. It is concluded that intrauterine ethanol exposure has effect on the development of vestibular, postural, sensory and motor coordination. The alterations in the amounts of iron and copper which are important cofactors for certain neurotransmitters and enzymes in the brain could play role in the neurobehavioural deficits observed. Intrauterine ethanol ingestion affects development of sensory and motor reflexes as well as the amounts of iron and copper in both cerebrum and cerebellum in a reciprocal manner.
\end{abstract}

Keywords: ethanol, intrauterine, neurobehaviour, neurochemistry.

*Author for Correspondence

I. S El-ladan, A. Usman, DUJOPAS 7 (3b): 171-179 2021 


\section{INTRODUCTION}

There has been a long-standing role of alcohol consumption in culture and its role in social connectedness and relaxation. However, overconsumption has been linked to multitude of serious adverse health and societal problems including violence, unemployment and broken relationships (Dejong et al., 2019). Alcohol has been reported to have potency in inducing neuroteratogenicity (Sandra and Michael, 2003; Allam and Abdul-Hamid, 2013). Since 1973, it has become clear that exposure of otherwise normal human foetuses to high levels of alcohol damages a substantial number of the exposed neonates' brains in a variety of ways collectively referred to as the foetal alcohol spectrum disorders (FASDs). Often this damage can be seen directly in brain images obtained much later in life (Fred, 2006). Stratton et al., (1996) reported Alcohol Related Neurodevelopmental Disorder (ARND), an evidence of CNS abnormality (which include an abnormally small head, abnormal brain structures, and ARND neurological signs), evidence of a behavioural or cognitive disorder inconsistent with the expected developmental level, with hereditary factors, or with the environment, or both. One of the most devastating and extreme consequences of developmental alcohol exposure is the loss of neurons, and the data documenting neuronal loss are derived mostly from animal studies. Animal and clinical scientific investigations have revealed that ethanol diffuses through placenta and distributes rapidly into the foetal compartment, the ethanol has slower elimination time which leads to its accumulation in the amniotic fluid (Gupta et al., 2016).

Although selective programmed neuronal death is a normal aspect of CNS development, excessive neuronal death disrupts the development of normal neural networks and may lead to cognitive and behavioural dysfunctions (in both humans and animals). Distinct regions and specific cell types appear to be affected by alcohol-induced neuronal loss. Studies in rats have clearly demonstrated that alcohol causes a reduction of cerebellar Purkinje cells, cells of the olfactory bulb, and pyramidal cells in a part of the hippocampus known as the CA1 region (Livy et al., 2003).

Mammalian cortex is characterised by inside out cortical migration of new, late-produced cortical neurons moving toward the surface post the layers of already migrated cells (Rahimi-Balaei et al., 2018). The presence of the pyramidal cells and axons providing the principal output of the cortex characterised the cerebral cortex (Hellwig, 2000). The appearance and the maturation of a number of sensorimotor reflexes are components of the mature motor repertoire of the animal, and the expression of these can be correlated with the development and maturation of the nervous system (Cassidy et al., 1994).

The proper maintenance of the amounts of trace elements is very essential in health and disease in an organism, they are vital in proper functioning of nervous system (Takeda, 2004). They are involved in physiological and biochemical processes such as serving as cofactors for several enzymes and proteins. Many metals like iron $(\mathrm{Fe})$, copper $(\mathrm{Cu})$ or zinc (Zn) fulfil various essential biological functions and are thus vital for all living organisms. For instance, they play important roles in nervous system, participating in a wide range of processes such as neurotransmitter synthesis, myelination or synaptic transmission (Bohic et al., 2011). Copper and iron are transition elements essential for life. These metals are required to maintain the brain's biochemistry such that deficiency or excess of either copper or iron leads to diseases of the central nervous system. Alteration in copper and iron metabolism in the brain has been implicated in many neurodegenerative diseases, for example, copper and iron levels both show marked increase with age and may adversely interact with the A beta peptide causing its accumulation as well as production of neurotoxic hydrogen peroxide 
$\left(\mathrm{H}_{2} \mathrm{O}_{2}\right)$, thus, contributing to the pathogenesis of dementia of Alzheimer-type (DAT) (Swerdlow, 2007). Transferrin may be involved in the physiological transport and utilisation of iron and manganese into the brain. It is reported that the brain transferrin concentration is decreased in neurodegenerative diseases such as Alzheimer's disease and Parkinson's disease and that brain iron metabolism is also altered (Takeda, 2004).

The aim of this study is to determine the effects of intrauterine ethanol exposure on the levels of iron and copper in the cerebrum and cerebellum in neonatal Wistar rats and correlate with how the ethanol affects development of neurobehaviour in the study animals. This will help in elucidating many ways in which ethanol exerts its feototoxicity.

\section{MATERIALS AND METHOD}

\section{Experimental Animals}

Twenty one (21) Wistar rats were used for the experiment, comprising of 14 females and 7 males. The males weighed $(200 \mathrm{~g}-220 \mathrm{~g})$ while the females weighed $120 \mathrm{~g}-140 \mathrm{~g}$. Animals were housed in wire mesh cages under standard conditions and were maintained on standard rodent pellets (Hybrid Feeds ltd.) and water ad libitum. The animals were allowed to acclimatize for 2 weeks prior to commencement of the experiment. The study protocol was conducted in accordance with "Guide for the Care and Use of Laboratory Animals" (National Research Council, 1996).

\section{Cycling of the Animals}

Oestrous cycle in Wistar rats last 4-5 days and consists of four main oestrous phases namely: pro-oestrous, oestrous, metoestrous and dioestrous. These phases are characterised by the morphology of cells found in the vaginal smear. The pro-oestrous phase is predominantly composed of nucleated epithelial cells, the oestrous precedes the pro-oestrous and it is predominated by irregular anucleated cornified cells, whereas the metoestous preceding the oestrous is composed of three different types of cells, these are: nucleated epithelial cells, irregular anucleated cornified cells, and leucocytes, then the last phase which is di-oestrous is predominated by leucocytes (Adebisi, 2009). The Oestrous phase determination was conducted according to Marcondes et al., (2002)

\section{Ethanol Administration}

Absolute ethanol was obtained from Tricura GmbH and Co., Germany (CAS no. 17-7), it was diluted with distilled water to make $20 \%$ and $30 \% \mathrm{v} / \mathrm{v}$. the dosage administered were $0.5 \mathrm{ml}$ of $20 \% \mathrm{v} / \mathrm{v}$ and $30 \% \mathrm{v} / \mathrm{v}$ via gavage following the method of Adebisi, (2009) and Allam \& Abdulhamid (2013).

\section{Experimental Design}

Following confirmation of mating and pregnancy, the dams were randomly divided into seven (7) groups, each group comprising of two (2) female rats. Group A served as the control which received only distilled water throughout the gestation period, Groups B, C and $\mathrm{D}$ received $0.5 \mathrm{ml}$ of $20 \%$ ethanol for 7,14 and 20 days during pregnancy respectively, while Groups E, F and $\mathrm{G}$ received $0.5 \mathrm{ml}$ of $30 \%$ ethanol for 7, 14 and 20 days during pregnancy accordingly.

\section{Neurobehavioural Tests \\ Surface Righting Reflex}

This test was conducted to measure the development of the vestibular reflex and motor coordination. It assesses the ability of the pups to co-ordinate the necessary movement to roll 
over from its back onto its paws. The rats were placed in supine position and the time taken in 30seconds to turn over to the prone position and place all four paws in contact with the surface was recorded. Each animal was tested in 2 trials on post-natal day five (PND 5). The maximum time allowed per trial was 30secs (Kihara et al., 2001).

\section{Cliff Avoidance}

On PND 6, the pup's ability to avert a cliff was assessed. Pups were placed on the end of a 5$\mathrm{cm}$-high platform with their noses and fore-digits suspended over the edge of the simulated cliff. The latency to back away from the edge of the cliff, turn, and crawl away was measured. This test is considered an evaluation of sensory and motor coordination development. Positive responses were noted when pups completed the task in $<30$ seconds. Each pup was tested in 1 trial (Kihara et al., 2001).

\section{Negative Geotaxis}

This test was conducted on PND 7 to assess motor co-ordination of the pups when challenged on a sloped surface. Latency to rotate the body is considered a measurement of vestibular and postural reflexes. The animals were placed with their nose pointed downward on an inclined grid $\left(45^{\circ}\right)$ of $30 \mathrm{~cm}$. The hindlimbs of the pups were placed in the middle of the grid. The time taken to complete a $180^{\circ}$ turn was measured. Each animal was given one trial at a maximum of 60seconds (Kihara et al., 2001).

\section{Atomic Absorption Spectrophotometry (AAS) of trace elements}

Varian Fast Sequential Atomic Absorption Spectrophotometry (model number AA240FS) was used to quantify the levels of iron and copper in both the cerebrum and cerebellum of the neonatal Wistar rats. The tissues were homogenised in phosphate buffer saline (PBS) prior to the meta-analysis. The homogenate was analysed using standard AAS protocol (EHD, 1995).

\section{Statistical Analysis}

The data was analysed using SPSS software version 20. The data were expressed as mean \pm SEM (Standard Error of Mean). One-Way Analysis of Variance (ANOVA) was used to compare the mean difference between and within the groups and a p-value of $<0.05$ was considered to be statistically significant. Bonferroni corrected post-hoc test was used to determine where the level of significance lies.

\section{RESULT}

Pre-weaning Neurobehavioural tests

Surface Righting Reflex

The control group A showed a quick reflex latency of 5.9secs when compared with the ethanol-treated groups especially groups B (12.2secs), F (14.3secs) and G (33.7secs) although the difference was not statistically significant. Groups C (6.0secs), D (5.3secs) and E (3.3secs) showed relatively close timing response compared to the control group, this also however was not statistically significant (Fig. 1)

\section{Cliff Avoidance}

The Control Group A, showed a quick latency of 9.9secs in avoiding the cliff compared to ethanol-treated Groups B (60secs), E (42.7secs), F (38.1secs), and G (29.9secs), though only Groups B and E were statistically significant ( $<<0.001$ and $p<0.05$ respectively) as shown in Fig. 1. 


\section{Negative Geotaxis}

Following the negative geotaxis test, Group A showed significant difference in the timing response compared to the ethanol-treated Groups E [51.5 secs $(p<0.05)], F$ [57.5secs $(p<0.001)]$ and $G$ [52.3secs $(<0.01)]$ as shown in fig. 1. The control group A also showed a quick reflex latency of 14.9 secs when compared with treated Groups B (23secs), C (26.9secs) and D (37.7secs), although not to statistical levels.

Trace Element meta-analysis using Atomic Absorption Spectrophotometry (AAS)

The result of the trace element analysis using AAS in both the cerebrum and cerebellum of the neonatal rats showed the presence of copper $(\mathrm{Cu})$ and iron $(\mathrm{Fe})$, with the concentration of iron being the highest $(\geq 10 \mathrm{mg} / \mathrm{kg})$ in both the tissues. The depletion in the amount of iron in the cerebrum of the treated groups was not statistically significant. On the other hand, the elevation in concentration of iron observed in the cerebellum of the treated groups was statistically significant with Groups $\mathrm{C}(\mathrm{P}<0.001), \mathrm{D}(\mathrm{P}<0.01)$ and $\mathrm{E}(\mathrm{P}<0.01)$ in comparison with the Group A (fig. 3).

Moreover, the obselved depletion in the amount of Copper in the cerebrum of the treated groups only met statistical significance with *kroup B $(0.5 \mathrm{ml} 20 \%$ ethanol 7 days $)$. Interestingly, there was statistical significant elevation in the amount of copper in the cerebellum of Groups D $(\mathrm{P}<0.01), \mathrm{E}(\mathrm{P}<0.05)$ and $\mathrm{F}(\mathrm{P}<0.001)$ when compared to the Control Group (fig. 3).

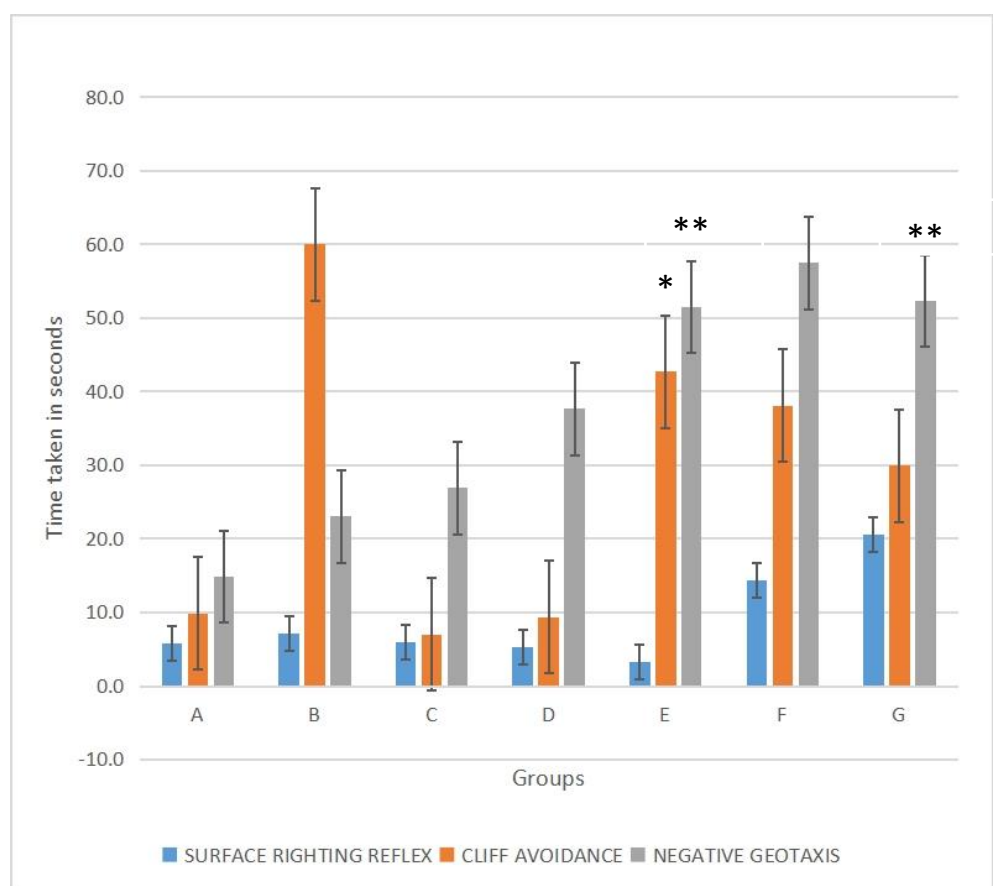

Figure 1: Comparison of the effects of intrauterine ethanol exposure on neuro-behaviour of the pups. ${ }^{*} \mathrm{P}<$ $0.05 ;{ }^{* *} \mathrm{P}<0.01 ;{ }^{* * *} \mathrm{P}<0.001$ 


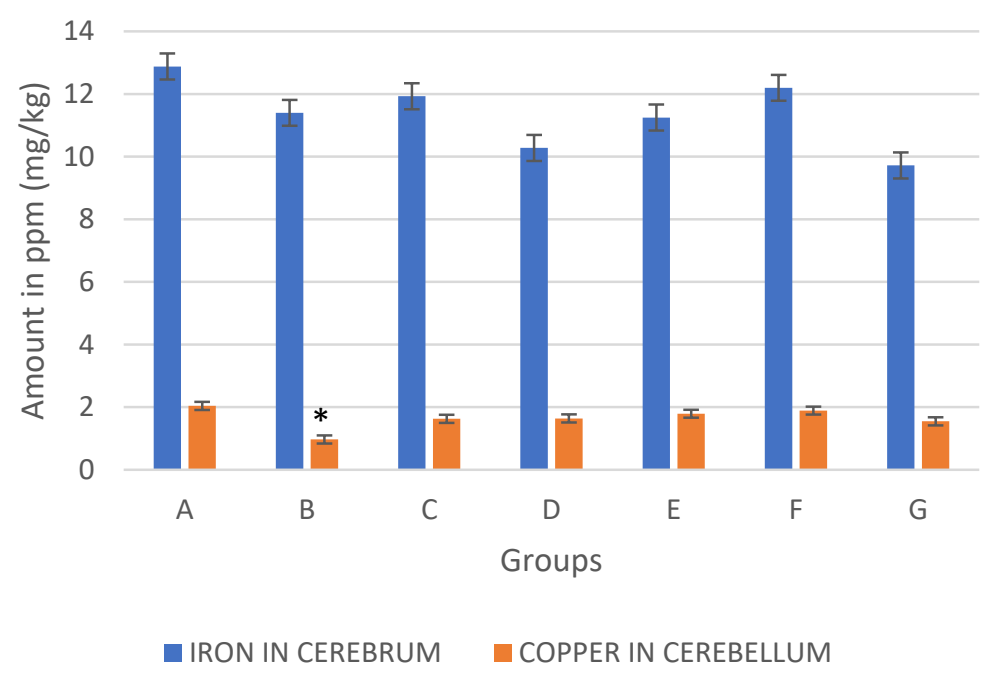

Figure 2. Comparison of the mean concentration of some trace elements in Cerebrum of neonatal Wistar rats. ${ }^{*} \mathrm{P}<0.05$

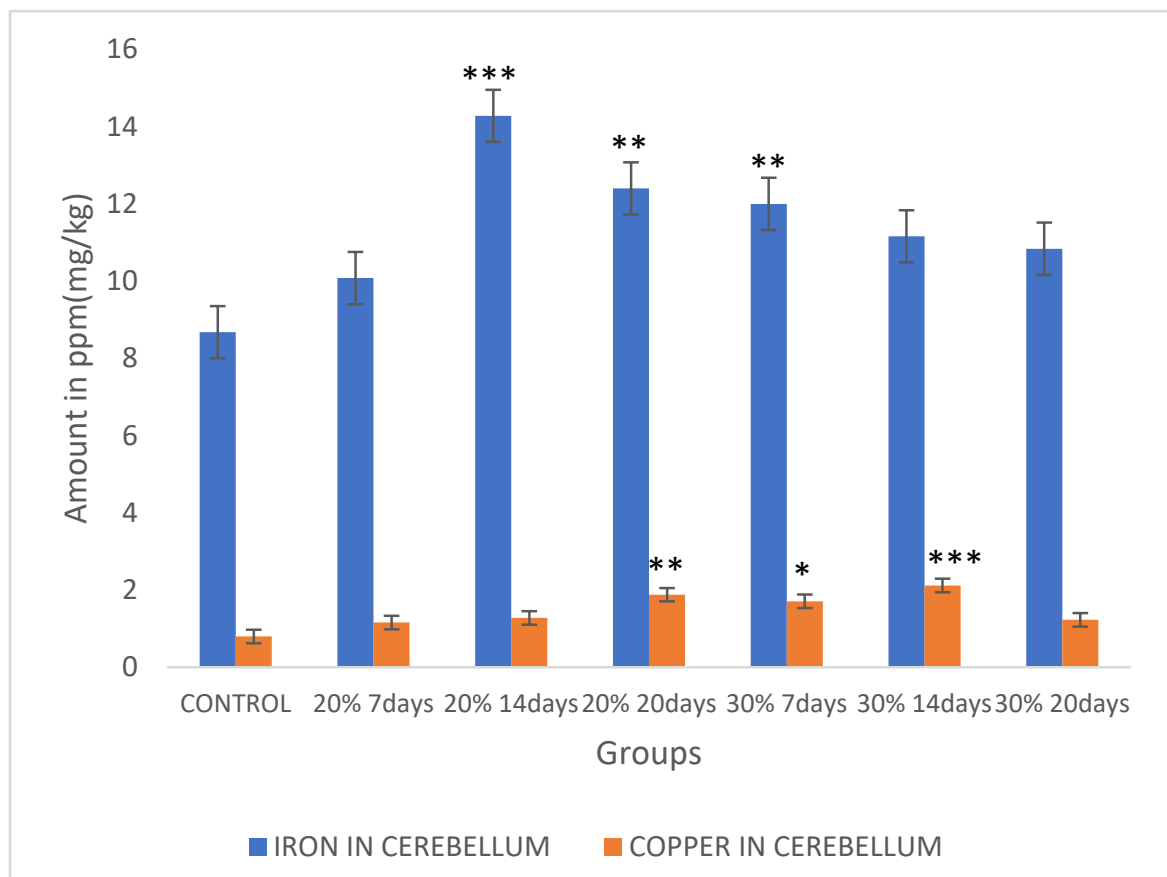

Figure 3: Comparison of the mean concentration of some trace elements in Cerebellum of neonatal Wistar rats. ${ }^{*} \mathrm{P}<0.05,{ }^{* *} \mathrm{P}<0.01,{ }^{* * *} \mathrm{P}<0.001$

\section{DISCUSSION}

The present study shows that intrauterine ethanol exposure has effect on the development of vestibular and postural reflexes, sensory and motor coordination of the neonates. The control group demonstrated quick latency in responding to developmental milestones assessing the sensory and motor reflexes, thus maternal ethanol ingestion affects the development of the reflexes. This agrees with the work of Allam and Abdulhamid (2013) who reported that ethanol ingestion during pregnancy leads to deficit in development of sensory and motor reflexes in the affected neonates. 
Iron and copper play vital role in the synthesis of numerous neurotransmitters as well as serving as co-factors for several enzymes and proteins. In the brain, iron and copper are important cofactors for enzymes involved in the neurotransmitter synthesis and myelin formation (Gaggeli, 2006 and Lutsenko, 2010). The uptake mechanism of the two metals interact, resulting to an interplay between the two metals (Tina et al., 2012), in essence, this could be the possible reason for the simultaneous reciprocal relationship of Iron and Copper elevation and depletion in cerebellum and cerebrum of the ethanol treated neonates respectively. Consequently, the effects observed in the trace elements could lead to deficit in neurotransmitter synthesis, thus affecting nervous coordination and activity of certain enzymes such as superoxide dismutase which play role in preventing the generation of free radicals in the brain tissue which could ultimately lead to the generation of reactive oxygen species (ROS) in the brain leading to neurodegeneration (Tina et al., 2012).

Both shortage and excess of iron and/or copper can affect the brain by affecting either neurotransmitter and/or protein synthesis (Tina et al., 2012). When in excess, they both participate in the fenton reaction which is a series of chemical reactions initiated by transition metals and hydrogen peroxide that leads to the formation of unstable free radicals that significantly affect DNA, proteins, and lipids, thereby causing deleterious damage to the cells (RiveraMancia et al., 2010). Therefore a strict regulation of these metals is vital for normal cellular function and survival. Copper is essential for normal CNS function, it is required as a cofactor for dopamine beta monooxygenase, peptidylglycine alpha amidating monooxygenase, superoxide dismutase, and a large number of other enzymes. Furthermore, it is required for mitochondrial respiration and oxidation of Iron, leaving the brain vulnerable in case it gets deprived of copper. Elevated copper observed in this study is toxic to the brain and thus it can cause accumulation that could result to symptoms such as dystonia and dysarthria as reported by Tina et al., (2012), who reported that copper accumulation was observed in Wilson disease resulting to Parkinsonian symptoms.

Excess copper in the brain is also reported to have excitotoxicity to dopaminergic neurons (Tina et al., 2012). Therefore, the spike in copper levels observed in the cerebellum of the ethanol treated neonates can lead to excitotoxicity of dopamine system in the cerebellum resulting in interference with the neuromodulatory effect of copper in the dopaminergic neurons which are important in motor function activity can be said to be compromised leading to effect on motor coordination as observed in the neurodevelopmental milestones in this study. Furthermore, the interplay between iron and copper metabolism in the brain as reported by Tina et al., (2012) could be inferred to be the possible reason for the effect on motor activity of the pups observed in the study.

\section{CONCLUSION}

The mechanism through which ethanol exerts its foeto-toxicity resulting to a reciprocal elevation and depletion in iron and copper in cerebellum and cerebrum respectively is poorly understood. It can be concluded that intrauterine ethanol ingestion at different phases of embryonic life affects the development of sensory, motor, vestibular and postural reflexes. It also affects the amount of iron and copper in both the cerebrum and cerebellum, thus playing role in the development of ethanol foeto-toxicity.

\section{ACKNOWLEDGEMENT}

The authors acknowledge the contribution of Professor S.S Adebisi and Dr W. O Hamman from Ahmadu Bello University Zaria, and Professor Austine O. Ibegbu from Alex Ekwueme 
Federal University, Ndufu-Alike for their guidance and constructive criticism in designing and carrying out the study.

\section{REFERENCES}

Adebisi, S. S. (2009). The toxicity of artesunate on bone development: the Wistar rat animal model of malaria treatment. Internet Journal of Parasitic Diseases. Vol. 4 (1).

Allam, A. A and Abdul-Hamid, M. (2013). Effect of Ethanol Ingestion in the Pregnant Albino Rat on The Development of Pyramidal Neurons. Life Science Journal, Vol.10 (3rd ed.), Pp.1760-1771

Bohic S, Ghersi-Egea JF, Gibon J, Paoletti P, Arnaud J, et al., (2011) Rôles biologiques des éléments traces dans le cerveau--exemples du Zn et du Fe [Biological roles of trace elements in the brain with special focus on Zn and Fe]. Rev Neurol (Paris). ;167(4):269-79. French. doi: 10.1016/j.neurol.2010.07.035. Epub 2010 Nov 5. PMID: 21056442.

Cassidy G, Boudrias D, Pflieger JF, Cabana T. (1994). The development of sensorimotor reflexes in the Brazilian opossum (Monodelphis domestica). Brain Behavioural Evolution. 43: 244-253

Chen, S., Mulgrew B, and Grant P.M (1993). "A clustering technique for digital communications channel equalization using radial basis function networks," IEEE Trans. on Neural Networks, vol. 4, pp. 570-578

Dejong K., Amy O. and Jamie O.L (2019). Alcohol Use in Pregnancy Clinical Obstetrics and Gynecology Vol. 62, No.1, 142-155

Duncombe J.U. (1959) "Infrared navigation-Part I: An assessment of feasibility," IEEE Trans. Electron Devices, vol. ED-11, pp. 34-39.

EHD (1995). Environmental Health Department, Canada. Determination of metals in biofluids and tissues: Sample preparation methods for atomic spectroscopic techniques. Spectromichica. (59): 291-319

Fred, L. B., Streissguth, P. C. and Sampson, P. (2006). Damage to the Human Cerebellum from Prenatal Alcohol Exposure: The Anatomy of a Simple Biometrical Explanation. The anatomical record (part b: new anatomy.) 289b: 195-209.

Gaggelli, E., Kozlowski, H., Valensin, D., and Valensin, G. (2006). Copper homeostasis and neurodegenerative disorders (Alzheimer's, prion, and Parkinson's diseases and amyotrophic lateral sclerosis). Chemical Review. 106: 1995-204410.1021/cr040410w [PubMed] [Cross Ref]

Gupta, Keshav \& Gupta, Vinay \& Shirasaka, Tomohiro. (2016). An Update on Fetal Alcohol Syndrome-Pathogenesis, Risks, and Treatment. Alcoholism: Clinical and Experimental Research. 40.10.1111/acer.13135.

Hellwig B. (2000). A quantitative analysis of the local connectivity between pyramidal neurons in layers 2/3 of the rat visual cortex. Biological Cybernetics, 82, pp. 111-121

Kandel, E. R., Schwartz, J. H. and Jessell, T. M. (2000). Principles of Neural Science. United State of America: McGraw-Hill. 4: 324. ISBN 0-8385-7701-6

Kihara, T., Tien, W. S., Takuya, M., Yoshiko, Y. and Takashi, T. (2001). Effects of the prenatal Rubratoxin-B exposure on behaviours of mouse offspring. Toxicological Science. 61: 368-373

Lin C.Y, Wu M, Bloom J. A., Cox I. J., and Miller M. (2001), "Rotation, scale, and translation resilient public watermarking for images," IEEE Trans. Image Process., vol. 10, no. 5, pp. 767-782. 
Livy, D. J., Miller, E. K., Maier, S. E and West JR (2003). Foetal alcohol exposure and temporal vulnerability: effects of binge-like alcohol exposure on the developing rat hippocampus. Neurotoxicological Teratology. 25(4): 447-58.

Lutsenko, S., Bhattacharjee, A., and Hubbard, A. L. (2010). Copper handling machinery of the brain. Metallomics. 2: 596-608 10.1039/c0mt00006j [PubMed] [Cross Ref]

National Research Council. 1996. Guide for the Care and Use of Laboratory Animals. Washington, DC: The National Academies Press. https:// doi.org/10.17226/5140.

Marcondes, F. K., Bianchi, F. J. and Tanno, A. P. (2002). Determination of the oestrous cycle phases of rats: some helpful considerations. Brazilian Journal of Biology. 62(4A): 609614

Rahimi-Balaei, M., Bergen, H., Kong, J., \& Marzban, H. (2018). Neuronal Migration During Development of the Cerebellum. Frontiers in cellular neuroscience, 12, 484. https:/ / doi.org/10.3389/fncel.2018.00484

RiveraMancia, S., PerezNeri, I., Rios, C., TristanLopez, L., RiveraEspinosa, L.et al., (2010). The transition metals Copper and Iron in neurodegenerative diseases. Chem. Biological Interaction. 186: 184-19910.1016/j.cbi.2010.04.010 [PubMed] [Cross Ref]

Sandra, M. M. and Michael, W. M. (2003). Ethanol induced neuronal death in organotypic cultures of rat cerebral cortex. Developmental Brain Research. 147: 135-141.

Shipp, S. (2007). "Structure and function of the cerebral cortex". Current Biology 17(12): 443449

Stratton, K., Howe, C., and Battaglia, F. (1996) Institute of Medicine. Summary. Fetal Alcohol Syndrome: Diagnosis, Epidemiology, Prevention, and Treatment, Washington, DC: National Academy Press.

Swerdlow R. H. (2007). Pathogenesis of Alzheimer's disease. Clinical interventions in aging, 2(3), 347-359.

TAKEDA Atsushi (2004). YAKUGAKU ZASSHI (Journal of the Pharmaceutical Society of Japan) 124(9): 577-585, 2004

Tina, S., Lisbeth B. M. and Torben M. (2012). Impairment of Interrelated Iron and Copper Homeostatic Mechanisms in Brain Contributes to the Pathogenesis of Neurodegenerative Disorders. Frontiers in Pharmacology. (3): 169 